\title{
Characterization of Implanted Stents through Neointimal Tissue Bioimpedance Simulations
}

\author{
J. M. Portillo-Anaya, P. Pérez, Student Member, IEEE, G. Huertas, A. \\ Olmo, J. A. Serrano, A. Maldonado-Jacobi and A. Yúfera, Member, IEEE
}

\begin{abstract}
This work describes how is possible the definition of the light hole or lumen in implanted stents affected by restenosis processes using the BioImpedance (BI) as biomarker. The main approach is based on the fact that neointimal tissues implied in restenosis can be detected and measured thanks to their respective conductivity and dielectric properties. For this goal, it is proposed a four-electrode setup for bioimpedance measurement. The influence of the several involved tissues in restenosis: fat, muscle, fiber, endothelium and blood, have been studied at several frequencies, validating the setup and illustrating the sensitivity of each one. Finally, a real example using a standard stent, has been analyzed for stable and vulnerable plaques in restenosis test cases, demonstrating that the proposed method is useful for the stent obstruction test. Bioimpedance simulation test has been performed using the electric physics module in COMSOL Multiphysics®.
\end{abstract}

\section{INTRODUCTION}

Ischemic heart disease is the leading cause of morbidity and mortality in the world $[1,2]$. The interior of the elastic and muscular arteries shrinks (stenosis), mainly by the growth of a lipid layer (atherosclerosis), causing angina chest pain and acute myocardial infarction. There are certain regions which are susceptible to this, such as the left anterior descending (LAD) coronary [3]. Among the existing treatments, the most popular one employs a flexible metal mesh (stent) to mechanically support the opening of stenotic segments and ensure permeability [4-5]. However, despite improvements on the surgery techniques and the stent design [6], there is a high probability of medium-term in-stent restenosis [7], Figure 1.

Characterizing the neointima tissue in real time would ease the integration of a readout circuits to measure and compensate the sensitivity loss of the MEMS [8,9]. Different publications have recently emerged that using bioimpedance techniques to follow up the restenosis by studying the growth of new tissue on the artery (neointimal layer). Some solutions provide the catheter with a matrix of microelectrodes $[10,11]$. Other works place the electrodes straightaway on the stent [12]. It is also proposed to use the stent itself as a big electrode [13]. These alternatives still need catheterization to connect external measurement equipment, though.

A published method monitors cell-cultures in vivo based on their bioimpedance [14]. It consists on converting the CellCulture Under Test (CCUT) into a "biological oscillator"

Reaseach supported by the Cátedra de Telefónica Inteligencia en la Red (0399), and Junta de Andalucía grant for research groups, 2017/TIC-178.

A. Yúfera is with the Instituto de Microelectrónica de Sevilla/Universidad de Sevilla (IMSE/US) Av. Americo Vespuccio 28, 41092, Sevilla, Spain. (corresponding author phone:+34954466666; e-mail: yufera@imsecnm.us.es) whose characteristic parameters (i.e., frequency, amplitude, phase, etc.) are related with cell-culture evolution and can be easily determined. This approach, known as Oscillation-Based Test (OBT), is also valid to monitor the neointimal tissue growth [19]. A closed system topology imposes a quasisinusoidal signal to stimulate the tissue, and the input resistor keeps the current injected to the blood vessel within safety margins. The relevant parameters are extracted from oscillations: frequency and amplitude.

This paper is primarily focused on the development of a setup for neointimal tissue characterization using finite element simulations to measure its bioimpedance, and secondly, the application of this setup to different involved tissues to check the sensitivity of each one, including a study case with a stent, characterizing its lumen in stable and vulnerable plaques in restenosis situations. Section II describes how the finite element simulations are performed and the tissue impedances measured. In section III it is defined the electrode set-up for optimum bioimpedance test. Simulations for the several involved tissues are illustrated in section IV, together with the results obtained for a stent case. Section V highlights some points of the presented work.

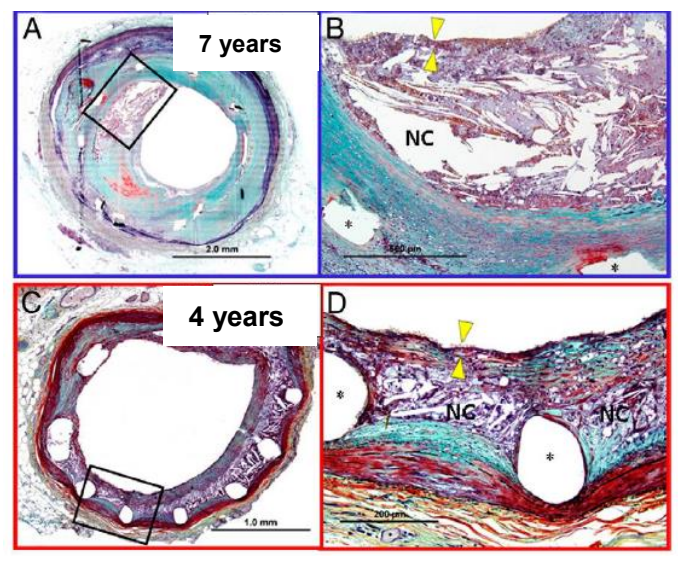

Figure1. Restenosis in implated bare-metal stent (BMS) after 7 years (A) and restenosis in implanted drug-eluting stent (DES) after 4 years (C), from [6]. White gaps around lumen in images (A) and (C) correspond to stent.

(B) and (D) are zoom images from (A) and (C) respectively. Yellow arrowheads in (B) and (D) point to thin fibrous layers.

J. M. Portillo-Anaya is with the Universidad de Sevilla, ETSII Av. Reina Mercedes sn, 41012 Sevilla, Spain. He is now with the Department of Tecnología Electrónica, Universidad de Sevilla, (email: portilloanaya@gmail.com).

P. Pérez, G. Huertas, A. Olmo, J. A. Serrano, A. Maldonado are with the Instituto de Microelectrónica de Sevilla/ Universidad de Sevilla (IMSE/US) (e-mail: \{pablopg, gloria, aolmo, serrano, maldonado\}@imse-cnm.us.es). 


\section{Finite ELEMENT SimULATION}

\section{A. Simulation Tool}

COMSOL Multiphysics ${ }^{\circledR}$ represents a standard tool for Finite Element Analysis (FEA) and simulations of structures, to evaluate some physics over them. In our case, for a given tissue, an $\mathrm{AC}$ electric current of $3 \mu \mathrm{A}$ amplitude is applied between two electrodes, and the voltage response is measured by other two high impedance nodes $[15,16]$. The voltage-tocurrent ratio (BI) is measured at each frequency, obtaining its magnitude and phase.

\section{B. Tissue model}

The neointimal tissue is composed by several layers: fat, muscle, fiber, endothelium and blood. Each one has its own electrical properties: conductivity $(\sigma)$ and permittivity $(\varepsilon)$ [17]. Besides, both properties are dependent on frequency and are introduced as simulation parameters for FEA $[18,19]$. FEA is done for several tissues types and sizes.

Table 1: Electric conductivities (S/m)

\begin{tabular}{|c|c|c|c|c|c|}
\hline Freq. (Hz) & Fat & Muscle & Fiber & Endothel. & Blood \\
\hline $10^{0}$ & 0.035 & 0.202 & 0.25 & 0.05 & 0.700 \\
\hline $10^{1}$ & 0.038 & 0.202 & 0.25 & 0.05 & 0.700 \\
\hline $10^{2}$ & 0.041 & 0.267 & 0.25 & 0.05 & 0.700 \\
\hline $10^{3}$ & 0.042 & 0.321 & 0.25 & 0.05 & 0.700 \\
\hline $10^{4}$ & 0.043 & 0.341 & 0.25 & 0.05 & 0.700 \\
\hline $10^{5}$ & 0.043 & 0.362 & 0.3 & 0.0502 & 0.702 \\
\hline $10^{6}$ & 0.044 & 0.503 & 0.35 & 0.146 & 0.840 \\
\hline
\end{tabular}

Table II: Relative permittivities.

\begin{tabular}{|c|c|c|c|c|c|}
\hline Freq.(Hz) & Fat & Muscle & Fiber & Endothel. & Blood \\
\hline $10^{0}$ & $9.9110^{6}$ & $2.6210^{7}$ & $210^{4}$ & $3.6110^{3}$ & $5.2610^{3}$ \\
\hline $10^{1}$ & $5.0310^{6}$ & $2.5710^{7}$ & $210^{4}$ & $3.6110^{3}$ & $5.2610^{3}$ \\
\hline $10^{2}$ & $1.5210^{5}$ & $9.3310^{6}$ & $110^{4}$ & $3.6110^{3}$ & $5.2610^{3}$ \\
\hline $10^{3}$ & $1.9310^{4}$ & $4.3510^{5}$ & $110^{3}$ & $3.6110^{3}$ & $5.2610^{3}$ \\
\hline $10^{4}$ & $9.1210^{2}$ & $2.5910^{4}$ & $110^{2}$ & $3.6110^{3}$ & $5.2610^{3}$ \\
\hline $10^{5}$ & $1.0110^{2}$ & $8.0910^{3}$ & $210^{1}$ & $3.5810^{3}$ & $5.2210^{3}$ \\
\hline $10^{6}$ & $5.0810^{1}$ & $1.8410^{3}$ & $110^{1}$ & $1.8510^{3}$ & $3.1210^{3}$ \\
\hline
\end{tabular}

\section{Electrode SET-UP}

Bioimpedance is commonly measured through 2 or 4 electrodes setups. The main advantages of the 4-electrodes one is that voltage response is not dependent from the electrode model, when is measured by a high-input-impedance amplifier. In this case, a 4-wires system is selected for measuring the tissue or biological layers. 2 for current excitation and 2 for voltage response measure. Several configurations have been analyzed for this. It was selected the one shown in Figure 2 as it makes the current lines travel parallel, reducing in this way the leakages. Generally, the more parallel the current lines are, the better sensitivity the voltage measurement electrodes are. This only happens when the emitter electrodes face measurement electrodes and vice versa, as it can be seen at top view in Figure 2. Setup dimensions were selected to adapt it to an average LAD diameter [20]. Gold electrodes were set to $7 \mu \mathrm{mx} 7 \mu \mathrm{m} \times 2.5 \mu \mathrm{m}$ size. The frequency for FEA was ranged from $1 \mathrm{~Hz}$ until $1 \mathrm{MHz}$, being the current excitation amplitude of $3 \mu \mathrm{A}$.

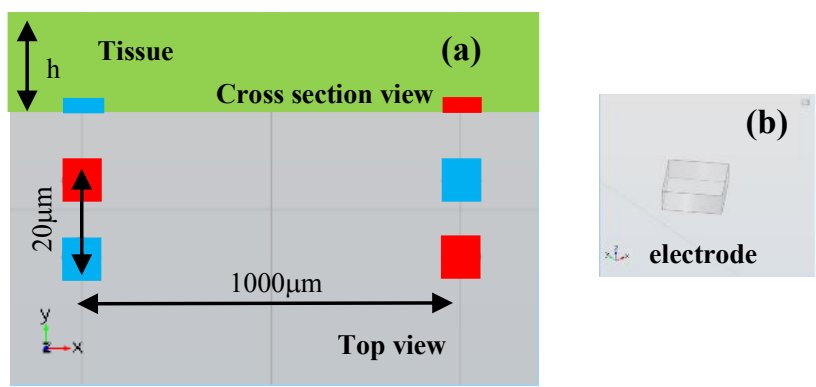

Figure 2. (a) Four-electrode setup placed above a insulating layer. Two electrodes are for current excitation (blue) and two-electrodes for BI measure (red). (b) Gold electrode $(7 \mu \mathrm{mx} 7 \mu \mathrm{mx} 2.5 \mu \mathrm{m})$. $\mathrm{h}$ is the tissue thickness.

\section{EXPERIMENTAL FEA BIOIMPEDANCE}

FEA were performed using COMSOL Multiphysics ${ }^{\circledR}$ computer program. We consider four types of simulations for the characterization of the neointimal tissue.

\section{A. Single tissue:}

Changes in each tissue thickness, and the frequency are modified respectively. A parametric sweep has been done for every single tissue in each simulation, taking as variable the thickness (h in Fig. 2) of a prism made by the respective tissues. It changed from 25 to $200 \mu \mathrm{m}$ with a step of $25 \mu \mathrm{m}$, while the prism depth and width were constant: 20 and $2 \mathrm{~mm}$ respectively. As regards the electrode set-up, were $1 \mathrm{~mm}$ and $20 \mu \mathrm{m}$ apart. Once data were collected and ordered, magnitude and phase impedances were depicted to show their respective variations due to tissue thickness and frequency changes.

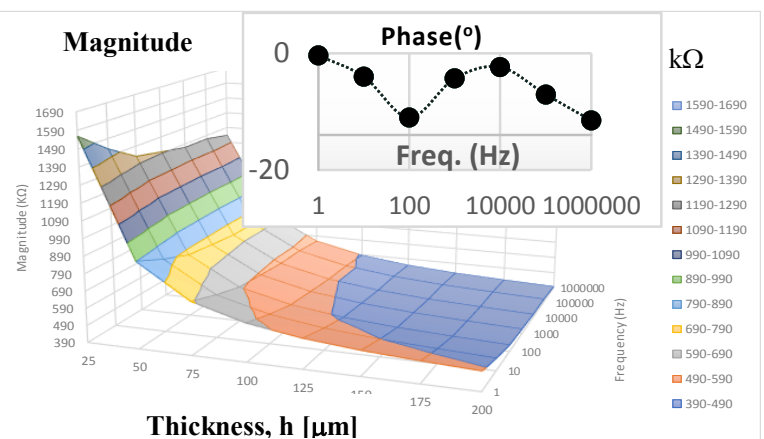

Figure 3. Magnitude and phase impedance in fat layer.

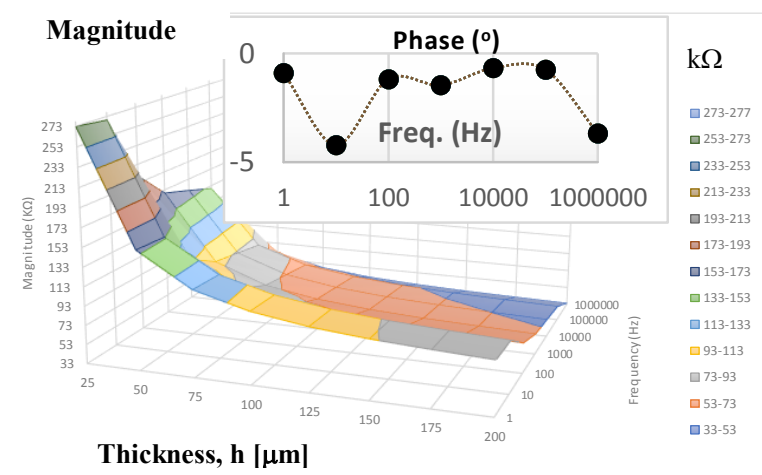

Figure 4. Magnitude and phase impedance in muscle layer. 


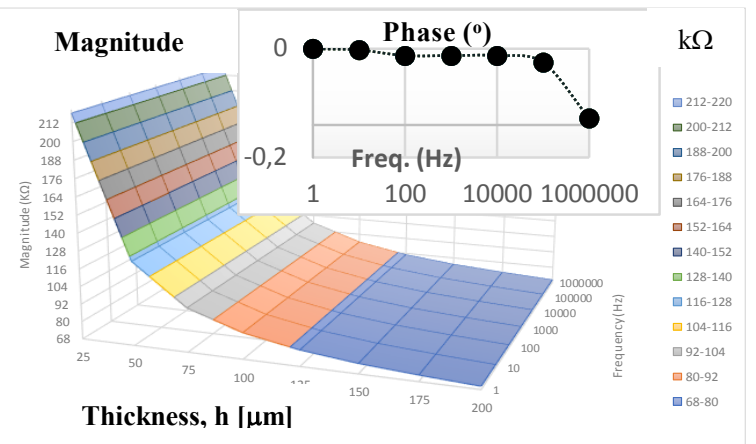

Figure 5. Magnitude and phase impedance in fiber layer.

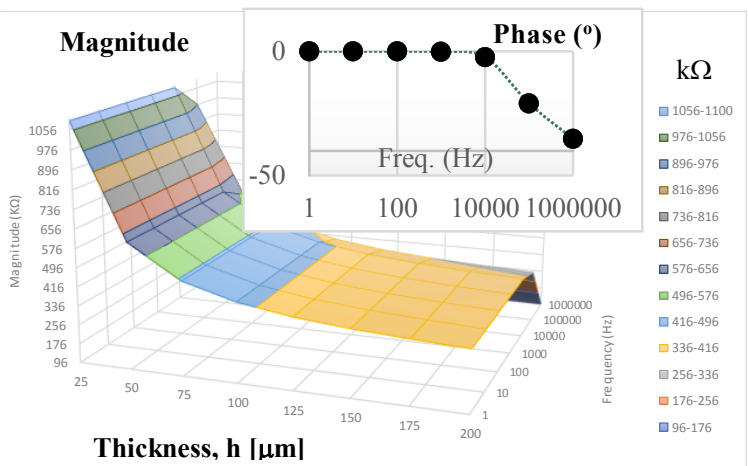

Figure 6. Magnitude and phase impedance in endothelium layer.

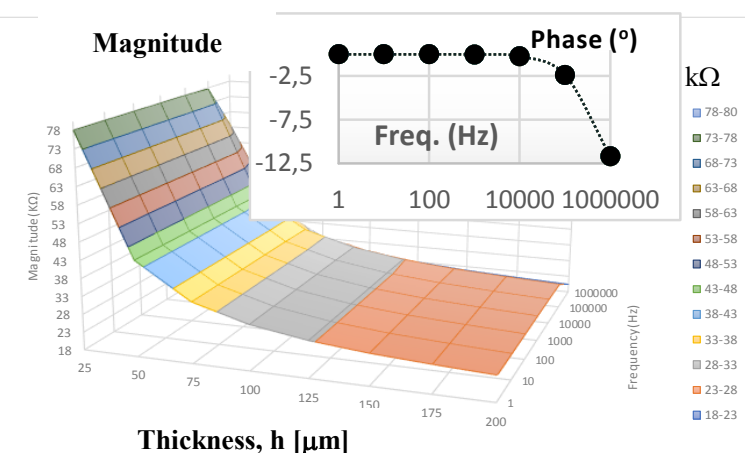

Figure 7. Magnitude and phase impedance in blood layer.

\section{B. Restenosis tissues:}

It is illustrated how impedance depends on the tissues thickness and the frequency respectively. The differences between this and previous section are that in this one, all tissues were taken into account at the same time, and the thickness changes differently, as it can be checked in Table III. Here, it has been tried to represent a supposed coronary lumen getting narrower. For this reason, it has been supposed that the vessel radius was $1150 \mu \mathrm{m}$. Nine different cases have been simulated with the increasing of the occlusion percentage. Figures 8 and 9 depict how impedance varies when frequency changes and the vessel occlusion is increased respectively. In Figure 9, it can be appreciated a good sensitivity to occlusion increments. Phase changes are low, and graphics are omitted for the limited paper space.
TABLE III: Case definitions for FEA restenosis inside stents considering several tissues involved in the neointimal layer, and the resulting Occlusion Percentage calculated for a stent of $1150 \mu \mathrm{m}$ of internal radius.

\begin{tabular}{|c|c|c|c|c|c|c|}
\hline & \multicolumn{7}{|c|}{ Thickness (in $\boldsymbol{\mu m}$ ) } & Occlusion \\
\hline Case & Fat & Muscle & Fiber & Endothelium & Blood & (\%) \\
\hline 1 & 0 & 25 & 0 & 10 & 1115 & 3 \\
\hline 2 & 0 & 50 & 0 & 10 & 1090 & 5 \\
\hline 3 & 25 & 50 & 5 & 10 & 1060 & 8 \\
\hline 4 & 50 & 100 & 10 & 10 & 980 & 15 \\
\hline 5 & 100 & 100 & 20 & 10 & 920 & 20 \\
\hline 6 & 200 & 100 & 40 & 10 & 800 & 30 \\
\hline 7 & 250 & 175 & 75 & 10 & 640 & 44 \\
\hline 8 & 300 & 200 & 100 & 10 & 540 & 53 \\
\hline 9 & 400 & 300 & 100 & 10 & 340 & 70 \\
\hline
\end{tabular}

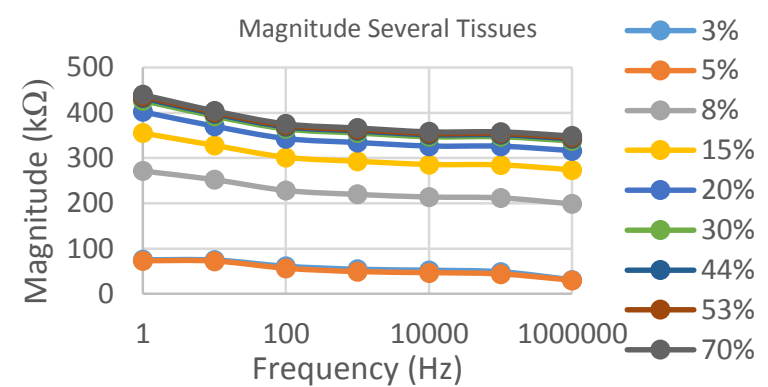

Figure 8. Bioimpedance magnitude variation due to frequency changes for several occlusion values.

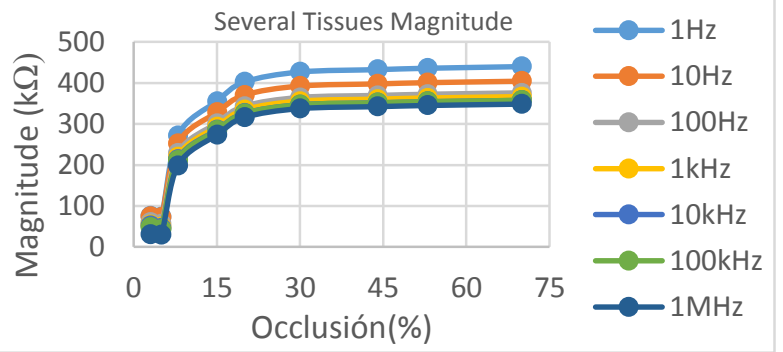

Figure 9. Bioimpedance magnitude variation due to occlusion increments for several frequencies.

\section{Symmetrical restenosis}

Impedance changes are analyzed when tissue thickness and the frequency are modified respectively on real stents. In this case, the geometry is totally different regarding the previous sections: A stent is placed around the tissues which are concentric to it. The eight sets of two electrodes (emitter and measurement electrodes respectively), each of them placed on every octagon vertex in Figure 10, are approximately the same apart as the above sections. This octagon represents the stent end as it was seen from a lateral view. The tissues growth rate follows the Table III patterns.
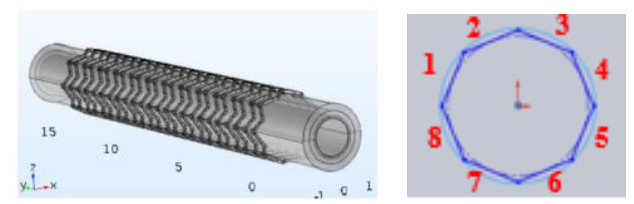

Figure 10. Regular geometry stent, and the 8 sets of four-electrodes placed around and inside the stent contour. The internal radius considered for the stent is of $1150 \mu \mathrm{m}$. 
The corresponding graphics for magnitude and phase represent how impedance varies when the vessel occlusion is increased and the frequency changes. The eight setups must measure the same impedance in a symmetrical geometry and like before section so Figures 8 and 9 show a similar impedance.

\section{Non-symmetrical restenosis:}

This situation implements the impedance response when restenosis is not symmetrical respect to tissues, as it happens in case $\mathrm{C}$. The considered electrode setup is illustrated in Figure 11(a), with the corresponding asymmetrical lumen. This case has been considered as a possible restenosis situation. The tissue profiles shown in Figures 11(b) and 11(c) corresponds to muscle and fat tissue increments respectively. The fifth and sixth set of four-electrodes shown in Figure 11(a) have always been taken as a reference because the corresponding portion has always been incremented in Figure $11(b)$ and 11(c) the same as in case C.

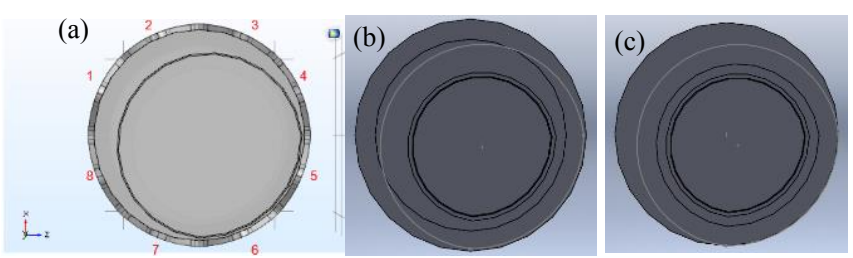

Figure 11. General irregular lumen inside a stent (a), generated by the tissue muscle increments (b) or fat tissue increments (c).

For the irregular lumen in Figure 11(a), the nine cases in Table III were simulated, in the same frequency range. Figure 12 shows the magnitude response obtained at the 8 electrodepairs, at $1 \mathrm{kHz}$ frequency, considering irregular and regular lumens. It has been considered a stable plaque case (muscle increases), Figure 12 (blue), and a vulnerable [21] plaque (fat increases), Figure 12 (black), respect to the reference (symmetrical lumen), in the case 6 in Table III, Figure 12 (red). It can be appreciated how the impedance magnitude measured at the test point (1-8) decreases for lower sizes of fat, while increases for similar changes in muscle. Also, variation ranges in fat impedance magnitude are larger than in muscle ones. This impedance magnitude that increases due to fat tissue increments represents a potential biomarker to detect vulnerable plaques in restenosis cases.

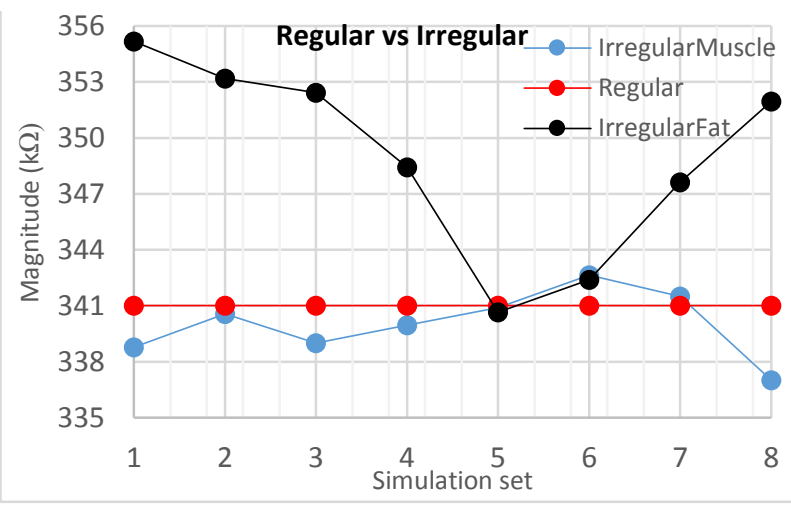

Figure 12. Magnitude measure using FEA in regular and irregular lumen inside a stent at $1 \mathrm{kHz}$. Regular (red) corresponds to case 6 in Table III. Irregular lumen is provoked by (blue) muscle increment (stable plaque), and (black) fat tissue increment (vulnerable plaque).

\section{CONCLUSIONS}

This work presents a progress into the FEA applied to neointimal characterization in implanted stents. It has been proposed a four electrodes setup that minimizes the electric current lines leakage for BI measurements. The dependence on the several tissues of neointimal layer are delivered for magnitude and phase, together to the analysis of symmetrical and asymmetrical lumens examples. Our preliminary results demonstrate that it is possible to distinguish vulnerable from stable plaque in restenosis cases by following the increment/decrement in magnitude impedance at $1 \mathrm{kHz}$ frequency.

\section{REFERENCES}

[1] OECD/EU, Health at a Glance: Europe 2016: State of Health in the EU Cycle, OECD Publishing, Paris. 2016.

[2] N. Townsend, L. Wilson, P. Bhatnagar, K. Wickramasinghe, M. Rayner, M. Nichols. "Cardiovascular disease in Europe: epidemiological update,” Eur Heart J2016; 37 (42): 3232-3245. 2016.

[3] D. L. Kasper, et al, Eds., Harrison's Principles of Internal Medicine, 19th Edition. New York: McGraw Hill, 2016.

[4] T. J. Gundert et al,"Identification of Hemodynamically Optimal Coronary Stent Designs Based on Vessel Caliber,"TBME 59(7) 2012.

[5] E. Chow, A. Chlebowsky, S. Chakraborty, W. Chappell, P. Irazoqui, "Fully Wireless Implantable Cardiovascular Pressure Monitor Integrated with a Medical Stent," TBME. 57 (6): 1487-1496. 2010.

[6] G. W. Stone et al., "Comparison of a polymer-based PaclitaxerlEluting Stent with a Bare Metal Stent in patients with complex Coronary Artery Disease," J. Amer. Medical Assoc., 294 (10), 2005.

[7] S-J. Park et al., "In-stent neoatherosclerosis - a final common pathway of late stent failure," J. Amer. College Cardiology, 59 (23), 2012.

[8] J.A. Miguel, et al.,"A novel computer-assisted design tool for implantable MEMS pressure sensors," Microprocessors and Microsystems, 46(A): 75-83. 2016.

[9] D. Rivas-Marchena et al., "Energy-efficient implantable transmitter for restenosis monitoring with intelligent-stents," in $37^{\text {th }}$ Int. C. IEEE Eng. Medicine Biology Soc. EMBS, pp:3323-3326. 2015.

[10] T. Süselbeck et al., "In vivo intravascular electric impedance spectroscopy using a new catheter with integrated microelectrodes," Basic Research Cardiology, 100, (1): 28-34, 2005.

[11] F. Yang and R. Patterson, "A novel impedance-based tomography approach for stenotic plaque detection - a simulation study," Int. J. Cardiology, 144 (2): 279-283, 2010.

[12] N. L. Opie et al., "Chronic impedance spectroscopy of an endovascular stent-electrode array," J. Neural Eng., 13, (4) 2016.

[13] L. Shedden et al., "Towards a self-reporting coronary artery stent measuring neointimal growth associated with in-stent restenosis using electrical impedance techniques," Biosensors \& Bioelec.,26 (2), 2010.

[14] G. Huertas et al., "The bio-oscillator: a circuit for cell-culture assays," IEEE TCAS-II, 62, (2):164-168, 2015.

[15] H. P. Schwan and C. D. Ferris, "Four-electrode null techniques for impedance measurement with high resolution," Review of Scientific Instruments, 39, (4): 481-485, 1968.

[16] S. Grimnes and Ø. G. Martinsen, Bioimpedance and Bioelectricity Basics, 3rd Ed.: Elsevier, 2014.

[17] C. Gabriel and S. Gabriel. (1996, Jun.) Compilation of the dielectric properties of body tissues at RF and microwave frequencies. [Online]. Available: http://niremf.ifac.cnr.it/docs/DIELECTRIC/Report.html

[18] A. Olmo and A. Yúfera, "Computer simulation of microelectrode based bio-impedance measurements with Comsol," in Biodevices. pp: 178-182. 2010.

[19] D. Rivas, et al, "Real-Time Electrical Bioimpedance Characterization of Neointimal Tissue for Stent Applications," Sensors. 17, (8). 2017.

[20] J. T. Dodge, et al, "Lumen diameter of normal human coronary arteries. Influence of age, sex, anatomic variation, and left ventricular hypertrophy or dilation", Circulation, 86 (1): 232-246, jul. 1992.

[21] S. Christodoulos, et al., "Coronary Atherosclerotic Vulnerable Plaque: Current Perspectives", J. Am. Heart Assoc., 6 (3) p. e005543. 